\title{
Propofol Infusion Syndrome: A Case Report
}

\author{
Oleksa Rewa ${ }^{\mathrm{a}, \mathrm{e}}$, John Muscedere ${ }^{\mathrm{b}, \mathrm{c}, \mathrm{d}}$
}

\begin{abstract}
Propofol is an ultra-short acting intravenous sedative/hypnotic agent commonly used for sedation in the intensive care unit (ICU) and operating room (OR). Up until recently, no severe systemic side effects were apparent but more recently severe and potentially lethal side effects have been sporadically reported and termed propofol-infusion syndrome (PRIS). Herein we describe a case of a 23 years old previously healthy male admitted to the ICU secondary to trauma. Due to excessive agitation and failure of other agents, he was sedated on high dose propofol ( $>100 \mu \mathrm{g} / \mathrm{Kg} / \mathrm{min}$ ) for several days. Following this he developed rhabdomyolysis, worsening renal failure, hyperkalemia, refractory acidosis, and eventually expired secondary to cardiovascular collapse. PRIS was attributed as the cause of death. Since its introduction in the 1980s propofol has been widely used for sedation in the ICU. PRIS has become an increasingly recognized phenomenon where propofol infusions have been used for prolonged periods of time. It is characterized by progressive rhabdomyolysis, renal failure, hyperkalemia, metabolic acidosis, Brugada-like pattern changes on ECG, cardiovascular collapse and finally death, all characteristics present in our patient. Several important risk factors for this condition exist such as prolonged propofol infusion, young age, head trauma, airway infection, increased catecholamine and glucocorticoid levels and malnutrition. The mechanism of PRIS remains incompletely understood but it is thought to be due to mitochondrial dysfunction with propofol serving to uncouple the respiratory chain; as such treatment involves stopping the medication and supportive care. In the vast majority of patients, propofol is safe with few side effects but given its potential for lethal complications heightened vigilance is required for its continued use. In particular, to minimize the potential for the development of PRIS, dose limitations and daily re-eval-
\end{abstract}

\footnotetext{
Manuscript accepted for publication May, 22, 2011

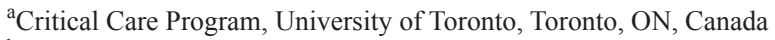

bepartment of Medicine, Queen's University, Kingston, ON, Canada

'Critical Care Program, Queen's University, Kingston, ON, Canada

${ }^{\mathrm{d} C}$ Clinical Evaluation Research Unit, Kingston General Hospital,

Kingston, ON Canada

${ }^{\text {e}}$ Corresponding author: Oleksa Rewa, 80 John Street Suite 3410,

Toronto, Ontario, M5V 3X4, Canada. Email: oleksa.rewa@utoronto.ca
}

doi: http://dx.doi.org/10.4021/jmc1269w uation as to the necessity of its continued use are required. Further, PRIS must be considered when patients develop unexplained organ dysfunction with metabolic acidosis, increased creatinine phosphokinase and hemodynamic instability. Awareness of the early clinical manifestations of PRIS leading to prompt termination of propofol infusion and appropriate supportive care may improve its outcome if it does develop.

Keywords: Propofol infusion; Syndrome; Trauma

\section{Introduction}

Propofol (2,6-diisopropylphenol) is an ultra-short acting

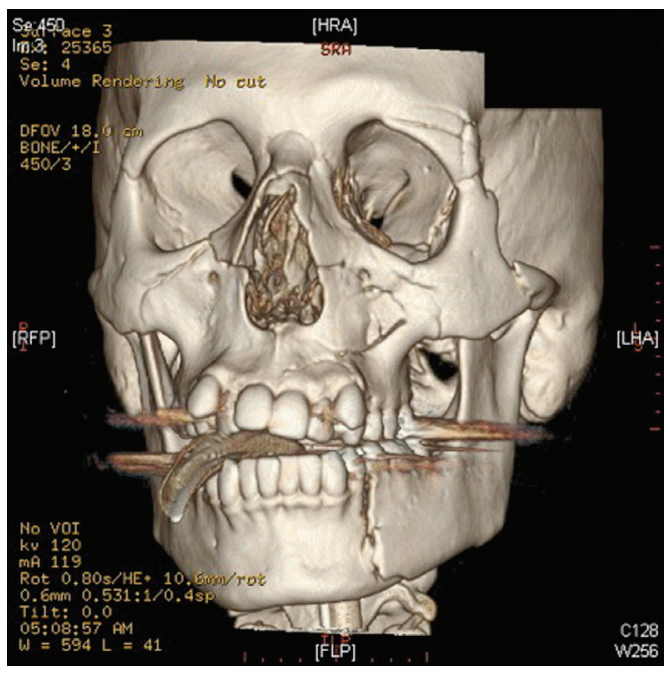

Figure 1. Depicted above is a CT reconstruction of the patient's facial injuries. These include fractures of the bilateral medial and lateral pterygoid plates, bilateral nasal bones, and left zygoma. Comminuted fractures of the medial and lateral walls of both maxillary sinuses are visualized. There are fractures of the medial and lateral walls of the left orbit, which is comminuted in the lateral wall. There are also bilateral orbital floor fractures, which involve both orbital rims and infra-orbital foramina. There are also several fracture lines extending from the left maxillary sinus into the left maxilla. There is also a fracture through the left mandibular body. 


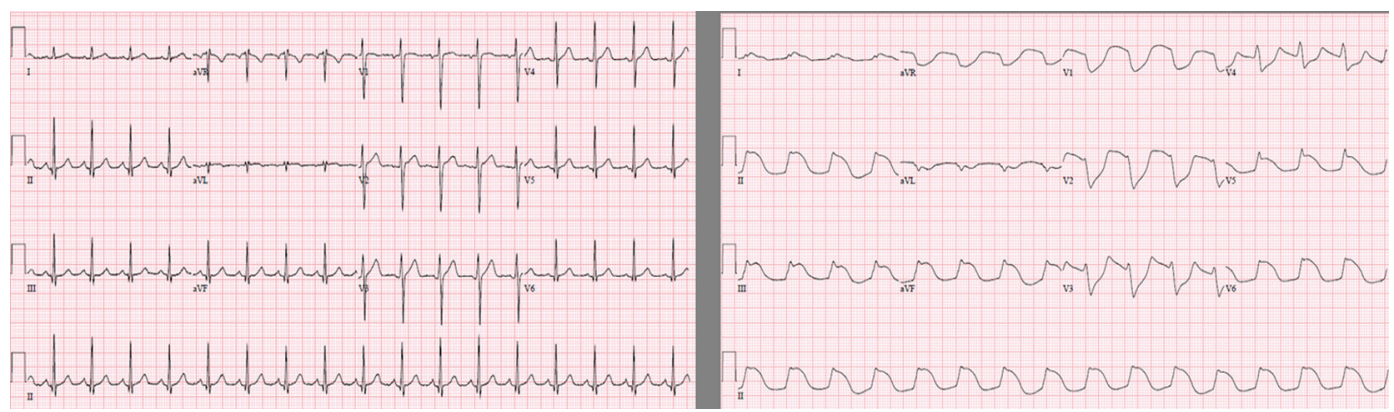

Figure 2. These 2 electrocardiograms were taken on ICU day 1 and day 4. Notable are new ST elevations in leads V1-V3 in a Brugada-like pattern often seen in patients with propofol-infusion syndrome.

intravenous sedative/hypnotic agent commonly used for sedation in the intensive care unit (ICU) and operating room (OR) [1]. Its exact mechanism of action is unknown, but it is postulated that propofol acts on both $\gamma$-aminobutyric acid (GABA) receptors directly and inhibits the N-methylD-aspartate (NMDA) receptor, modulates calcium influx through slow calcium-ion channels and acts as a powerful central nervous system depressant $[2,3]$. Propofol decreases cerebral blood flow, metabolic demands and acts as an anticonvulsive; as such it is commonly utilized as a sedative for ICU patients with traumatic brain injuries, status epilepticus, delirium tremens, status asthmaticus and sepsis [4]. Up until recently, known side effects of propofol were limited to dose related hypotension, cardio-respiratory depression, local skin irritation and hypertriglyceridemia with prolonged infusions (AstraZeneca 2005). However, severe and potentially lethal side effects have been sporadically reported which were first reported in children in the 1980s and termed propofol-infu- sion syndrome (PRIS) [5, 6]. This syndrome usually develops after periods of prolonged propofol infusion and is most typically seen in the ICU. Since it was first recognized, there have been periodic case reports in both pediatric and adult populations but PRIS often remains an unrecognized entity and its true incidence remains unknown. Herein, we describe an additional case.

\section{Case Report}

A 23 years old previously healthy young male was admitted to a tertiary care ICU secondary to a fall. Injuries included multiple facial lacerations, a zygoma fracture, left mandibular fracture, nasoethmoidal fracture, right parietal lobe contusion, and fractures of both forearms (Fig. 1). He was intubated due to decreased level of consciousness, agitation, combativeness, and for airway protection. Initial laboratory

Table 1. Worsening Rhabdomyolysis, Kidney Injury, Hyperkalemia and Metabolic Acidosis are Evident Throughout the Patients Stay all Indicative of Worsening PRIS

\begin{tabular}{|c|c|c|c|c|c|c|c|}
\hline & WBC & $\mathrm{Cr}$ & $\mathbf{K}$ & Trop I & CK & pH & aHCO3 \\
\hline Day 1 & 10.8 & 101 & 2.9 & & & 7.32 & 24 \\
\hline Day 2 & 7.0 & 89 & 3.3 & & & 7.39 & 26 \\
\hline Day 3 & 12.0 & 100 & 3.3 & & & 7.35 & 28 \\
\hline Day 4 & 6.9 & 92 & 3.2 & 0.218 & 973 & 7.37 & 27 \\
\hline Day 5 & 7.5 & 85 & 3.4 & 0.425 & 2428 & 7.36 & 26 \\
\hline Day 6 & 18.3 & 131 & 6.5 & 0.696 & 7743 & 7.20 & 15 \\
\hline Day 7 & 32.4 & 238 & 7.1 & 2.918 & 63007 & 7.20 & 10 \\
\hline
\end{tabular}


Table 2. Common Risk Factors and Typical Manifestations of PRIS

\begin{tabular}{ll}
\hline \multicolumn{1}{c}{ Risk Factors } & \multicolumn{1}{c}{ Manifestations } \\
\hline $\begin{array}{l}\text { Propofol administration for }>48 \mathrm{hrs} \mathrm{in} \\
\text { doses }>67 \mathrm{mcg} / \mathrm{kg} / \mathrm{min} \text { or } 5 \mathrm{mg} / \mathrm{hr}\end{array}$ & Rhabdomyolysis \\
Male sex & Renal Failure \\
Age $<19$ years old & Hyperkalemia \\
Head trauma & Lactic acidosis \\
Airway infection & Hypertriglyceridemia \\
Increased catecholamine and & Bradycardia and \\
glucocorticoid levels & Cardiovascular collapse \\
Malnutrition & \\
\hline
\end{tabular}

investigations were unremarkable other than an elevated blood alcohol level.

Initial sedation was with midazolam and fentanyl infusions but these were changed to a propofol infusion at 100 $\mu \mathrm{g} / \mathrm{kg} / \mathrm{min}$ to facilitate periodic neurological examinations. Boluses of fentanyl or morphine were administered for analgesia. He remained stable and was scheduled for operative repair of his facial injuries on ICU admission day 5, but on arrival to the OR became acutely unstable, requiring increased oxygen and vasopressors. He was brought back to the ICU requiring intermittent boluses of phenylephrine and was started on norepinephrine, epinephrine and vasopressin infusions. He was also found to have a new Brugada like pattern on his ECG (Fig. 2) and on echocardiography, a dilated and akinetic right ventricle was identified. Laboratory findings included leukocytosis, hyperkalemia, metabolic acidosis, evidence of new acute kidney injury and increased creatinine phosphokinase (Table 1). Further, he developed new runs of intermittent monomorphic ventricular tachycardia, and was begun on an amiodarone infusion. Chest x-ray at this time revealed a new airspace density in the right mid and lower lung zones and left perihilar region; a diagnosis of ventilator-associated pneumonia with severe sepsis with shock was entertained and appropriate antibiotic and supportive therapy were begun. Due to the echocardiography findings, anticoagulation for possible thrombo-embolic disease causing acute right ventricular failure was commenced. Renal replacement therapy was started and methylene blue and dexamethasone were administered due to his worsening hemodynamic status. On admission day 6 , a widening of his QRS developed which deteriorated into pulseless electrical activity cardiac arrest and he was unable to be resuscitated. Cardiovascular collapse secondary to prolonged propofol infusion was postulated as the cause of death. Due to difficulties in contacting the patient's family, our research ethics board was contacted and waived the requirement for written consent purposes of this case report (REB \#6006371).

\section{Discussion}

Propofol was first introduced as a sedative agent in the 1980s [7]. Since that time, its usage has become widespread due to its ultra-short half-life, minimal 'hang-over' effects, and up until recently, relatively benign side effect profile. However, since the late 1980s and early 1990s, PRIS has become an increasingly recognized phenomenon where propofol infusions have been used for prolonged periods.

PRIS is a complex condition involving progressive rhabdomyolysis, renal failure, hyperkalemia, metabolic acidosis, Brugada-like pattern change on ECG, cardiovascular collapse and finally death. Based on the published case reports to date, there have been several risk factors recognized for the development of PRIS. These include prolonged ( $>$ 48 hours) propofol administration in doses $>67 \mu \mathrm{g} / \mathrm{kg} / \mathrm{min}$, male gender, age $<19$ years old, head trauma, airway infection, increased catecholamine and glucocorticoid levels and malnutrition $[5,6]$. Our patient fulfilled several of these factors; most notably prolonged infusion of high dose propofol, male gender, head trauma and being suspected of having developed a ventilator-associated pneumonia. He also manifested many of the features of PRIS including rhabdomyolysis, renal failure, hyperkalemia, lactic acidosis, new Brugada-like ECG pattern and finally cardiovascular collapse (Table 2).

There currently exists no known treatment for PRIS other than early recognition of the syndrome, prompt cessation of the propofol infusion and supportive care. One of the reasons for this is that the exact mechanism of how PRIS manifests is not completely understood. The most current hypothesis 
involves mitochondrial dysfunction with propofol serving to uncouple the respiratory chain with resultant cellular hypoxia and multi-system organ dysfunction. It has also been postulated that propofol impairs fatty acid oxygenation leading to accumulation of free fatty acids and again, cellular hypoxia. Free fatty acids are pro-arrhythmogenic which may then lead to cardiovascular collapse. Other theories have focused on depletion on carbohydrate stores causing increased lipolysis and fat oxidation leading to secondary acidosis through accumulation of free fatty acids and ketones. Others still have postulated that perhaps it is not propofol itself causing this syndrome, but rather the disease state of the patient causing inadequate oxygen utilization and subsequent muscle necrosis leading to renal failure and metabolic acidosis. Histopathologic examinations of cardiac and skeletal muscle from patients suffering from PRIS reveal extensive myocytolysis and muscle necrosis [7]. Irrespective of the exact mechanism, the PRIS appears to involve an imbalance between tissue oxygen demand and supply leading to injury. Finally, it may not be a single mechanism that cause the manifestations of PRIS, but rather a cascade of events that 'prime' the individual and then others which 'trigger' the syndrome [6].

Since its introduction in the 1980s propofol has been widely used for sedation in the ICU. In the vast majority of patients, it is safe with few side effects but given its potential for lethal, albeit rare, complications heightened vigilance is required for its continued use. In particular, to minimize the potential for the development of PRIS, dose limitations and daily re-evaluation as to the necessity of its continued use are required. Further, PRIS must be considered when patients develop unexplained organ dysfunction with metabolic acidosis, increased creatinine phosphokinase and hemodynamic instability. Awareness of the early clinical manifestations of PRIS leading to prompt termination of propofol infusion and appropriate supportive care may improve its outcome if it does develop.

\section{Abbreviations}

WBC: White Blood Cells; Cr: Creatinine; K: Potassium; TropI: Troponin I; CK: Creatinine Phosphokinase; aHCO3: Arterial Bicarbonate

\section{References}

1. Kotani Y, Shimazawa M, Yoshimura S, Iwama T, Hara $\mathrm{H}$. The experimental and clinical pharmacology of propofol, an anesthetic agent with neuroprotective properties. CNS neuroscience \& therapeutics 2008;14:95106.

2. Ito Y, Izumi H, Sato M, Karita K, Iwatsuki N. Suppression of parasympathetic reflex vasodilatation in the lower lip of the cat by isoflurane, propofol, ketamine and pentobarbital: implications for mechanisms underlying the production of anaesthesia. British journal of anaesthesia 1998;81:563-8.

3. Zhan RZ, Qi S, Wu C, Fujihara H, Taga K, Shimoji K. Intravenous anesthetics differentially reduce neurotransmission damage caused by oxygen-glucose deprivation in rat hippocampal slices in correlation with N-methylD-aspartate receptor inhibition. Critical care medicine 2001;29:808-13.

4. De Cosmo G, Congedo E, Clemente A, Aceto P. Sedation in PACU: the role of propofol. Current drug targets 2005;6:741-4.

5. Fudickar A, Bein B. Propofol infusion syndrome: update of clinical manifestation and pathophysiology. Minerva anestesiologica 2009;75:339-44.

6. Kam PC, Cardone D. Propofol infusion syndrome. Anaesthesia 2007;62:690-701.

7. Diedrich DA, Brown DR. Analytic reviews: propofol infusion syndrome in the ICU. Journal of intensive care medicine 2011;26:59-72. 